Real Analysis Exchange

Vol. 24(2), 1998/9, pp. 703-728

Togo Nishiura, Department of Mathematics, Wayne State University, MI 48202, Department of Mathematics and Computer Science, Dickinson

College, Carlisle, PA 17013. e-mail: nishiura@dickinson.edu

\title{
ABSOLUTELY MEASURABLE FUNCTIONS ON MANIFOLDS
}

Dedicated to Casper Goffman on his 85 Th Birthday.

\begin{abstract}
The paper is an investigation of the collection of absolutely measurable functions defined on compact, connected manifolds. Several analytical properties of these functions defined on the manifold $I$, the unit interval of $\mathbb{R}$, have been studied by C. Goffman, D. Waterman and the author in Homeomorphisms in analysis [Math. Surveys Monogr., Number 54, American Mathematical Society, Providence, 1997]. It will be shown that these properties also hold for all compact, connected manifolds. The method of proof differs from those used earlier for the interval $I$. The key element here is the use of the von Neumann-UlamOxtoby Theorem for compact connected manifolds (proved here for the first time) which concerns measures induced by homeomorphisms.
\end{abstract}

The notion of absolutely measurable functions has appeared several times in the context of one-variable functions. (See the book [4] for some references and the book by J. C. Oxtoby [11] for related material on absolutely measurable sets.) A development of this notion in the context of manifolds is initiated here. A key element of this development is the von Neumann-Ulam-Oxtoby Theorem (see $[14,12,4]$ ). The symbol $\lambda_{n}$ will be reserved for the Lebesgue $n$-dimensional measure on $\mathbb{R}^{n}$.

The first section presents the preliminaries. Properties of a certain closurelike operator $\mathrm{P}$ in the context of compact, connected metric spaces are developed. Also a statement is given of the original von Neumann-Ulam-Oxtoby Theorem for intervals $I^{n}$ contained in $\mathbb{R}^{n}$. As all the measures encountered in

Key Words: absolutely measurable functions, absolutely measurable sets, universally measurable sets, compact manifolds

Mathematical Reviews subject classification: Primary 26B35, 28A05, 28A20, 58C05

Received by the editors August 1, 1998 
the paper are finite, the finiteness condition will not be stated in the discussion of measures except for the purpose of emphasis.

In the second section the von Neumann-Ulam-Oxtoby Theorem for compact, connected manifolds is proved.

The final section concerns Lebesgue's notion of equivalence. H. Lebesgue defined his notion of equivalence of functions in [10]. In an analogous manner, the notion of Lebesgue equivalence of measures can be made (see the first section). This notion is well suited for the von Neumann-Ulam-Oxtoby Theorem for manifolds. The theorem says that the Lebesgue equivalence classes of measures are determined by the value of the measure of the manifold. With the aid of the von Neumann-Ulam-Oxtoby Theorem for manifolds, three measuretheoretic theorems on manifolds will be proved. These theorems have been discussed for the manifold [0,1] in Chapters 2 and 11 of [4]. The proofs given there are somewhat different from those presented here. The first theorem is the equality $\mu$-almost everywhere of absolutely measurable functions to some Baire class 1 function where $\mu$ is some complete, nonatomic, Borel regular measure with the property that $\mu(U)>0$ whenever $U$ is a nonempty open set of the manifold. The second is a characterization of the absolute essential supremum of absolutely measurable functions. (The notion of absolute essential supremum is defined in the first section.) The third is a characterization of those functions defined on a manifold $M$ for which its composition with every self-homeomorphism of $M$ are in the space $\mathrm{L}^{1}(M, \mu)$, where $M$ is a fixed manifold and $\mu$ is a fixed complete, nonatomic, Borel regular measure with the property given above concerning open sets. Also a short discussion of product measures is included. The section ends with a discussion of Lebesgue equivalence of nonabsolutely measurable functions. In particular, a relationship between $\mathrm{L}^{1}(M, \mu)$ and $\mathrm{L}^{1}(M, \nu)$ is established, which yields a generalization of a theorem of T. Świątkowski $[13,9]$.

\section{Preliminaries}

The preliminary discussion is motivated by two useful properties of complete, nonatomic, Borel regular measures defined on a compact metric space. The first is that if $E$ is a $\mu$-measurable set with $\mu(E)>0$ then $E$ necessarily contains a nonempty perfect set because $\mu$ is nonatomic. The second is that, for each nonempty perfect set $E$ of a compact metric space, there is such a measure $\mu$ on the space with $\mu(E)>0$. For our purposes, only spaces whose cardinality exceeds $\aleph_{0}$ are of interest. Even more, the cardinality of each nonempty open set should exceed $\aleph_{0}$. These requirements are certainly met by compact metric spaces that are nondegenerate and connected. As usual, 
such a space is called a continuum.

The spaces $X$ in this section will always be assumed to be continua and the measures on $X$ will be complete, nonatomic and Borel regular. For a measure $\mu$ on $X$ and a $\mu$-measurable set $A$ of $X$, the measure $\mu\llcorner A$ on $X$ is defined by $(\mu\llcorner A)(E)=\mu(A \cap E)$ for $\mu$-measurable sets $E$, and the measure $\mu \mid A$ on $A$ is defined by $(\mu \mid A)(E)=\mu(E)$ for $\mu$-measurable subsets $E$ of $A$. The topological interior, closure and boundary of a subset $E$ of $X$ will be denoted respectively by $\operatorname{Int}_{X}(E), \mathrm{Cl}_{X}(E)$ and $\mathrm{Bd}_{X}(E)$.

A short discussion of the von Neumann-Ulam-Oxtoby Theorem will close the preliminary section.

\subsection{Absolutely Measurable Sets}

Let $\mathrm{M}(X)$ be the collection of all finite, complete, nonatomic, Borel regular measures on $X$ (note the inclusion of nonatomic). For each $\mu$ in $\mathrm{M}(X)$, the $\sigma$-algebra associated with $\mu$ is denoted by $\mathcal{A}(X, \mu)$. The natural definition for the collection of absolutely measurable sets of $X$ is

$$
\operatorname{ab} \mathcal{A}(X)=\bigcap\{\mathcal{A}(X, \mu): \mu \in \mathrm{M}(X)\} .
$$

Its subcollection

$$
\operatorname{ab} \mathcal{N}(X)=\{E: \mu(E)=0 \text { for every } \mu \text { in } \mathrm{M}(X)\}
$$

is called the collection of sets of absolute measure 0 . Clearly ab $\mathcal{A}(X)$ is a $\sigma$-algebra that contains the $\sigma$-algebra of Borel sets. A function $f$ defined on $X$ into a complete metric space is said to be absolutely measurable if it is measurable with respect to the $\sigma$-algebra $\operatorname{ab} \mathcal{A}(X)$; hence, a function is absolutely measurable if and only if it is measurable with respect to the $\sigma$-algebra $\mathcal{A}(X, \mu)$ for every $\mu$ in $\mathrm{M}(X)$. (Other authors have used the word universally instead of absolutely when $X=\mathbb{R}$.) The composition $g f$, where $g$ is a Borel measurable function, is absolutely measurable whenever $f$ is absolutely measurable on $X$. Moreover, the collection of all absolutely measurable real-valued functions on $X$ is a vector lattice that is closed under pointwise convergence of sequences. (The pointwise product of 2 real-valued functions $f$ and $g$ will be denoted by $f \cdot g$ since the usual juxtaposition convention has been used for composition.)

A measure $\mu$ in $\mathrm{M}(X)$ is said to be positive if $\mu(U)>0$ whenever $U$ is a nonempty open set (that is, the topological support of $\mu$ is $X$ ). Denote by $\mathrm{M}_{p}(X)$ the collection of all positive measures in $\mathrm{M}(X)$. Since $X$ is a continuum we have $\mathrm{M}_{p}(X) \neq \emptyset$. To see this, let $\left\{F_{i}: i=1,2, \ldots\right\}$ be a countable collection of nonempty, totally disconnected, perfect sets whose union is dense 
in $X$. Such a collection exists because $X$ is second countable. For each $i$ there is a homeomorphism $h_{i}: K \rightarrow F_{i}$ of the Cantor ternary set $K$ onto $F_{i}$. Let $\kappa$ be the measure on $K$ that is induced by the usual Cantor function. Then a measure $\kappa_{i}$ is induced on $X$ by the formula $\kappa_{i}(E)=\kappa\left(h_{i}{ }^{-1}[E]\right)$, where $E$ is a Borel subset of $X$. Clearly, $\sum_{i=1}^{\infty} 2^{-i} \kappa_{i} \in \mathrm{M}_{p}(X)$.

Finally, observe that, for each $\mu$ in $\mathrm{M}(X)$ and each $\nu$ in $\mathrm{M}_{p}(X)$, the measure $(1-\varepsilon) \mu+\varepsilon \nu$ is in $\mathrm{M}_{p}(X)$ whenever $0<\varepsilon<1$.

\subsection{The Closure-Like Operation P}

Let $X$ be a continuum. We define the closure-like operation $\mathrm{P}$ as follows: For a subset $E$ of $X$, the set $\mathrm{P}(E)$ consists of all points $x$ of $X$ with the property that $U \cap E$ contains a nonempty perfect set whenever $U$ is a neighborhood of $x$. Clearly, $E \subset F$ implies $\mathrm{P}(E) \subset \mathrm{P}(F)$. Obviously $\mathrm{P}(X)=X$, and $\mathrm{P}(E)$ is a closed set. Indeed, $\mathrm{P}(E)$ is a perfect set since $E \cap \mathrm{P}(E)$ is dense in $\mathrm{P}(E)$. Obviously, $\mathrm{P}^{2}(E)=\mathrm{P}(E)$. Also, $\mathrm{P}(E)=\emptyset$ for Borel sets $E$ precisely when $E$ is a countable set. There are absolutely measurable sets $E$ with cardinality exceeding $\aleph_{0}$ for which $\mathrm{P}(E)=\emptyset[11$, page 99].

We have the useful lemma:

Lemma 1.1. Let $X$ be a continuum. An absolutely measurable set $A$ will fail to be in $\operatorname{ab} \mathcal{N}(X)$ if and only if $A$ contains a nonempty perfect set. Consequently, if $Z$ is in $\operatorname{ab} \mathcal{A}(X)$, then $Z$ will be in $\operatorname{ab} \mathcal{N}(X)$ when and only when $\mathrm{P}(Z)=\emptyset$; whence $A \backslash \mathrm{P}(A) \in \operatorname{ab} \mathcal{N}(X)$ for every absolutely measurable set $A$. Finally, $\mathrm{P}\left(\operatorname{Int}_{X}(\mathrm{P}(A)) \cap A\right)=\mathrm{Cl}_{X}\left(\operatorname{Int}_{X} \mathrm{P}(A)\right)$ for every absolutely measurable set $A$.

Proof. Let $A$ be an absolutely measurable set with $\mu(A)>0$ for some measure $\mu$ in $\mathrm{M}(X)$. Since $\mu$ is Borel regular, there exists an uncountable Borel set contained in $A$. The first statement now follows.

The proofs of the remaining statements are left to the reader to supply.

Now the statement

$$
\mathrm{P}(E \cup F)=\mathrm{P}(E) \cup \mathrm{P}(F) \quad \text { whenever } E \text { and } F \text { are in ab } \mathcal{A}(X)
$$

follows from the lemma. Clearly $E \subset \mathrm{P}(E)$ may fail for some $E$, hence $\mathrm{P}$ is not the $\mathrm{Cl}_{X}$ operator.

We next establish a connection between absolutely measurable sets and Baire spaces. (A topological space $X$ is called a Baire space if the intersection of each countable collection of dense open sets is a dense subset of $X$.) 
Theorem 1.2. In a continuum $X$, suppose that $U$ is a nonempty Borel set with $U \subset \mathrm{P}(U)$. If $U$ is a Baire space and if $Z \in \operatorname{ab} \mathcal{N}(X)$, then $U \backslash Z$ is an uncountable Baire space.

Proof. Suppose that $U \backslash Z$ is countable. Then $U \subset \mathrm{P}((U \backslash Z) \cup(U \cap Z))=$ $\mathrm{P}(U \backslash Z) \cup \mathrm{P}(U \cap Z)=\emptyset$, a contradiction. Hence $U \backslash Z$ is uncountable.

Suppose that $U \backslash Z$ is not a Baire space. Then there is an open set $V$ of $X$ and there are, in the subspace $U \backslash Z$, closed and nowhere dense subsets $F_{i}$ of $U \backslash Z, i=1,2, \ldots$, that satisfy $V \cap(U \backslash Z)=\bigcup_{i=1}^{\infty}\left(V \cap F_{i}\right)$ and $V \cap(U \backslash Z) \neq \emptyset$. As $V$ is open in $X$ and $U \subset \mathrm{P}(U)$, we have the inclusion $V \cap U \subset \mathrm{P}(V \cap U)$. Since $V \cap U$ is a Borel set that is also a Baire space, there is no loss in assuming that $V \cap U$ is $U$. Clearly

$$
U \cap \mathrm{Cl}_{X}\left(F_{i}\right) \subset F_{i} \cup Z \text { and } U \backslash Z \subset \bigcup_{i=1}^{\infty} \mathrm{Cl}_{X}\left(F_{i}\right) .
$$

In the space $U$, for each $i$, we have that $U \cap \mathrm{Cl}_{X}\left(F_{i}\right)$ is closed and nowhere dense. The nowhere denseness follows because, in the space $U$, each nonempty open subset contains some nonempty open subset $W$ with $(W \backslash Z) \cap F_{i}=\emptyset$, whence $W \subset Z$. As $U \subset \mathrm{P}(U)$ yields $W \subset \mathrm{P}(W)$, we have the contradiction $\emptyset \neq W \subset \mathrm{P}(W) \subset \mathrm{P}(Z)=\emptyset$. As $U$ is a Baire space with $U \subset \mathrm{P}(U)$, the set $Y=U \backslash \bigcup_{i=1}^{\infty} \mathrm{Cl}_{X}\left(F_{i}\right)$ is uncountable. Since $Y$ is also a Borel set, $\mathrm{P}(Y) \neq \emptyset$ holds. We have

$$
Y=U \backslash \bigcup_{i=1}^{\infty} \mathrm{Cl}_{X}\left(F_{i}\right) \subset U \backslash(U \backslash Z) \subset Z,
$$

and the contradiction $\emptyset \neq \mathrm{P}(Y) \subset \mathrm{P}(Z)=\emptyset$ has been reached. Hence $U \backslash Z$ is a Baire space.

The above theorem was first proved for open sets $U$ of $[0,1]$ by A. M. Bruckner, R. O. Davies and C. Goffman in [1]. The next two lemmas are consequences of the theorem.

Lemma 1.3. Let $A_{i}, i=1,2, \ldots$, be a sequence of mutually disjoint, absolutely measurable sets of a continuum $X$ with $X=\bigcup_{i=1}^{\infty} A_{i}$. Then

$$
G_{i}=\operatorname{Int}_{X}\left(\mathrm{P}\left(A_{i}\right)\right) \backslash \bigcup_{j<i} \mathrm{P}\left(A_{j}\right), \quad i=1,2, \ldots,
$$

is a sequence of mutually disjoint open sets such that $\bigcup_{i=1}^{\infty} G_{i}$ is dense in $X$ and $\mathrm{P}\left(G_{i} \cap A_{i}\right)=\mathrm{Cl}_{X}\left(G_{i}\right)$ for each $i$.

Proof. Let $Z_{i}=A_{i} \backslash \mathrm{P}\left(A_{i}\right)$ for each $i$. Then $Z=\bigcup_{i=1}^{\infty} Z_{i} \in \operatorname{ab} \mathcal{N}(X)$. Observe

$$
X=\bigcup_{i=1}^{\infty} \mathrm{P}\left(A_{i}\right) \cup Z=\bigcup_{i=1}^{\infty}\left(\mathrm{P}\left(A_{i}\right) \backslash \bigcup_{j<i} \mathrm{P}\left(A_{j}\right)\right) \cup Z .
$$


The subspace $X \backslash Z$ is a Baire space by the above theorem, and $X \backslash Z$ is dense in $X$.

For each $i$ let $H_{i}=X \backslash \operatorname{Bd}_{X}\left(\mathrm{P}\left(A_{i}\right)\right)$. As $H_{i}$ is dense and open in $X$, we have that $H_{i} \backslash Z$ is dense and open in the subspace $X \backslash Z$. Hence the set

$$
(X \backslash Z) \cap \bigcap_{i=1}^{\infty} H_{i}=\bigcap_{i=1}^{\infty}\left(H_{i} \backslash Z\right)
$$

is dense in $X \backslash Z$ (and additionally is uncountable and a member of ab $\mathcal{A}(X)$ ). Since $\bigcap_{i=1}^{\infty} H_{i}=X \backslash \bigcup_{i=1}^{\infty} \operatorname{Bd}_{X}\left(\mathrm{P}\left(A_{i}\right)\right)$, we have

$$
\begin{aligned}
(X \backslash Z) \cap \bigcap_{i=1}^{\infty} H_{i} & =\left(\bigcup_{i=1}^{\infty}\left(\mathrm{P}\left(A_{i}\right) \backslash \bigcup_{j<i} \mathrm{P}\left(A_{j}\right)\right) \backslash Z\right) \backslash \bigcup_{i=1}^{\infty} \operatorname{Bd}_{X}\left(\mathrm{P}\left(A_{i}\right)\right) \\
& \subset\left(\bigcup_{i=1}^{\infty} G_{i}\right) \backslash Z \subset X \backslash Z .
\end{aligned}
$$

Hence $\left(\bigcup_{i=1}^{\infty} G_{i}\right) \backslash Z$ is dense in $X \backslash Z$ and the denseness of $\bigcup_{i=1}^{\infty} G_{i}$ in $X$ easily follows. Since $A_{i} \cap \operatorname{Int}_{X}\left(\mathrm{P}\left(A_{i}\right)\right)$ is dense in $\operatorname{Int}_{X}\left(\mathrm{P}\left(A_{i}\right)\right)$, the rest of the proof will follow.

The proof of the next lemma is a simple modification of (indeed, is contained in) the last lemma.

Lemma 1.4. Let $A_{i}, i=1,2, \ldots$, be a sequence of mutually disjoint, absolutely measurable sets of a continuum $X$ with $X=\bigcup_{i=1}^{\infty} \mathrm{P}\left(A_{i}\right)$. Then

$$
G_{i}=\operatorname{Int}_{X}\left(\mathrm{P}\left(A_{i}\right)\right) \backslash \bigcup_{j<i} \mathrm{P}\left(A_{j}\right), \quad i=1,2, \ldots,
$$

is a sequence of mutually disjoint open sets such that $\bigcup_{i=1}^{\infty} G_{i}$ is dense in $X$ and $\mathrm{P}\left(G_{i} \cap A_{i}\right)=\mathrm{Cl}_{X}\left(G_{i}\right)$ for each $i$.

Proof. The proof begins with the second line of the above proof with the set $Z$ replaced by the empty set.

Lemma 1.5. Let $A$ and $E_{i}, i=1,2, \ldots, N$, be absolutely measurable sets of a continuum $X$ such that $\mathrm{P}(A)=X$ and $X=\bigcup_{i=1}^{N} E_{i}$. Then there is a collection $G_{i}, i=1,2, \ldots, N$, of mutually disjoint, open sets whose union is dense in $X$ such that $\mathrm{P}\left(A \cap E_{i} \cap G_{i}\right)=\mathrm{Cl}_{X}\left(G_{i}\right)$ for each $i$, whence $\mathrm{P}\left(\bigcup_{i=1}^{N} A \cap E_{i} \cap G_{i}\right)=X$.

Proof. Let $A_{i}=A \cap E_{i}$ for each $i$. Then

$$
X=\mathrm{P}(A)=\mathrm{P}\left(A \cap \bigcup_{i=1}^{N} E_{i}\right)=\mathrm{P}\left(\bigcup_{i=1}^{N} A_{i}\right)=\bigcup_{i=1}^{N} \mathrm{P}\left(A_{i}\right) .
$$

Now apply the previous lemma. 
We now consider sequences of absolutely measurable real-valued functions. It will be convenient to use the sequence space $\mathbb{R}^{\infty}$ with the metric

$$
\rho(t, s)=\sum_{j=1}^{\infty} 2^{-j}\left|t_{j}-s_{j}\right|\left(1+\left|t_{j}-s_{j}\right|\right)^{-1},
$$

where the points $t$ of $\mathbb{R}^{\infty}$ are written in vector notation as

$$
t=\sum_{j=1}^{\infty} t_{j} e_{j}
$$

Hence, for $\sigma$-algebras $\mathcal{M}$ on $X$, a function $f: X \rightarrow \mathbb{R}^{\infty}$ is $\mathcal{M}$-measurable if and only if each of its coordinate functions $f_{j}: X \rightarrow \mathbb{R}$ is. Even more, $f$ will be in the Baire class $\alpha$ if and only if all of its coordinate functions $f_{j}$ are in that same Baire class.

In the vector space $\mathbb{R}^{\infty}$, the usual product $g f$ of $g: X \rightarrow \mathbb{R}$ and $f: X \rightarrow \mathbb{R}^{\infty}$ will have coordinates given by $(g f)_{j}=g \cdot f_{j}$. This juxtaposition notation should not be confused with the composition convention adopted earlier.

Proposition 1.6. For a continuum $X$, let $A$ be an absolutely measurable set with $\mathrm{P}(A) \neq \emptyset$ and let $f: X \rightarrow \mathbb{R}^{\infty}$ be an absolutely measurable function. Then there is a nonempty perfect set $F$ contained in $A$ such that $\chi_{F} f$ is in the Baire class 1.

Proof. There is a measure $\mu$ in $\mathrm{M}(X)$ such that $\mu(A)>0$. By Lusin's theorem, for each $j$, there is a closed set $F_{j}$ contained in $A$ such that $f_{j} \mid F_{j}$ is continuous and $\mu\left(F_{j}\right)>\left(1-4^{-j}\right) \mu(A)$. Obviously the closed set $\bigcap_{j=1}^{\infty} F_{j}$ contains a nonempty perfect set $F$ because it has positive $\mu$-measure and $\mu$ is nonatomic. That $\chi_{F} \cdot f_{j}$ is in the Baire class 1 easily follows from the Tietze extension theorem applied to $f_{j} \mid F$.

Lemma 1.7. Let $f: X \rightarrow \mathbb{R}^{\infty}$ be an absolutely measurable function defined on a continuum $X$ such that $f[X]$ is a countable set. Then there is an absolutely measurable set $E$ and there is a function $\varphi: X \rightarrow \mathbb{R}^{\infty}$ in the Baire class 1 such that $f|E=\varphi| E$ and $\mathrm{P}(E)=X$.

Proof. The sets $f^{-1}[y], y \in f[X]$, form a countable and mutually disjoint collection and the union of these sets is $X$. We apply Lemma 1.3 to get the mutually disjoint open sets $G_{y}, y \in f[X]$, whose union is dense in $X$ and satisfy $\mathrm{P}\left(f^{-1}[y] \cap G_{y}\right)=\mathrm{Cl}_{X}\left(G_{y}\right)$. Let $\varphi$ be the Baire class 1 function

$$
\varphi=\sum_{y \in f[X]} \chi_{G_{y}} y
$$

and let $E=\bigcup_{y \in f[X]} f^{-1}[y] \cap G_{y}$. As $\bigcup_{y \in f[X]} G_{y}$ is dense in $X$, we have $\mathrm{P}(E)=X$. An easy calculation leads to $f|E=\varphi| E$. 
We make a slight digression and consider functions that may not be absolutely measurable. Observe that if $E$ is any set then there is a Borel subset $B$ of $E$ with $\mathrm{P}(E)=\mathrm{P}(B)$ because $X$ is a second countable topological space.

Proposition 1.8. Let $f: X \rightarrow \mathbb{R}^{\infty}$ be a function defined on a continuum $X$ such that the image $f[X]$ contains a countable subset $Y$ with the property

$$
X=\bigcup_{y \in Y} \mathrm{P}\left(f^{-1}[y]\right) .
$$

Then there is a function $\varphi$ in the Baire class 1 and an absolutely measurable set $E$ such that $f|E=\varphi| E$ and $\mathrm{P}(E)=X$.

Proof. The proof is exactly the same as that of the previous lemma where Lemma 1.3 is replaced by Lemma 1.4.

We return to absolutely measurable functions. The next lemma concerns functions into $\mathbb{R}^{m}$ for finite $m$.

Lemma 1.9. For a continuum $X$ and $j=1,2, \ldots, m$, let $f_{1, j}$ be an absolutely measurable function and let $g_{1, j}$ be a function in the Baire class 1 with the property $\mathrm{P}\left(E_{1}\right)=X$ where $E_{1}=\bigcap_{j=1}^{m}\left\{x: f_{1, j}(x)=g_{1, j}(x)\right\}$.

If $f_{2, j}, j=1,2, \ldots, m$, are absolutely measurable functions such that the images of $f_{2, j}-f_{1, j}$ are contained in $\{0,1\}$, then there exists an absolutely measurable set $E_{2}$ contained in $E_{1}$ with $\mathrm{P}\left(E_{2}\right)=X$ and there are functions $g_{2, j}, j=1,2, \ldots, m$, in the Baire class 1 such that, for each $j$,

$$
g_{2, j}\left|E_{2}=f_{2, j}\right| E_{2} \quad \text { and } \quad\left(g_{2, j}-g_{1, j}\right)[X] \subset\{0,1\} .
$$

Proof. We use Lemma 1.5. In that lemma, let $A=E_{1}$ and use the finitely many sets $E_{y}=\bigcap_{j=1}^{m}\left(f_{2, j}-f_{1, j}\right)^{-1}\left[y_{j}\right]$, where $y=\left(y_{1}, y_{2}, \ldots, y_{m}\right)$ are in the product set $Y=\{0,1\}^{m}$. Then the open sets $G_{y}$ that result satisfy

$$
\mathrm{P}\left(\bigcup_{y \in Y}\left(E_{1} \cap E_{y} \cap G_{y}\right)\right)=X .
$$

To complete the proof, let

$$
g_{2, j}=\sum_{y \in Y} y_{j} \chi_{G_{y}}+g_{1, j} \quad \text { and } \quad E_{2}=\bigcup_{y \in Y}\left(E_{1} \cap E_{y} \cap G_{y}\right) .
$$

It follows easily that $f_{2, j}$ and $g_{2, j}$ agree on $E_{2}$.

Lemma 1.10. For a continuum $X$, let $f: X \rightarrow \mathbb{R}^{\infty}$ be an absolutely measurable function and let $\varphi: X \rightarrow \mathbb{R}^{\infty}$ be a function in the Baire class 1 with the property $\mathrm{P}(\{x: \rho(f(x), \varphi(x))<\varepsilon\})=X$ whenever $\varepsilon>0$. Then there is a function $g: X \rightarrow \mathbb{R}^{\infty}$ in the Baire class 1 such that

$$
\mathrm{P}\left(\bigcap_{j=1}^{\infty}\left\{x: f_{j}(x)=g_{j}(x)\right\}\right)=X .
$$


Proof. Let $U_{i}, i=1,2, \ldots$, be a basis for the open sets of $X$ and let

$$
E_{i}=\left\{x: \rho(f(x), \varphi(x))<2^{-i}\right\} \quad \text { for each } i .
$$

By Proposition 1.6 there is a nowhere dense, nonempty perfect set $F_{1}$ contained in $U_{1} \cap E_{1}$ such that $\left(f_{j}-\varphi_{j}\right) \cdot \chi_{F_{1}}$ is in the Baire class 1 for each $j$. Continuing inductively, we construct a sequence $F_{i}, i=1,2, \ldots$, of mutually disjoint, nowhere dense, nonempty perfect sets such that, for each $i$ and for each $j$,

$$
F_{i} \subset\left(U_{i} \cap E_{i}\right) \backslash \bigcup_{k<i} F_{k}, \quad\left(f_{j}-\varphi_{j}\right) \cdot \chi_{F_{i}} \text { is in the Baire class 1, }
$$

and, for each $i, \sup _{x \in X} \rho\left(\chi_{F_{i}}(x) f(x), \chi_{F_{i}}(x) \varphi(x)\right) \leq 2^{-i}$. Clearly, $\mathrm{P}(F)=X$ where $F=\bigcup_{i=1}^{\infty} F_{i}$. Uniform convergence implies that $g=\varphi+\sum_{i=1}^{\infty} \chi_{F_{i}}(f-$ $\varphi)$ is in the Baire class 1. As

$$
f-g=f-\varphi-\sum_{i=1}^{\infty} \chi_{F_{i}}(f-\varphi)=\chi_{X \backslash F}(f-\varphi),
$$

we have $F \subset\left\{x: f(x)_{j}=g_{j}(x)\right\}$ for every $j$, and the final equation in the lemma follows easily.

Theorem 1.11. Let $X$ be a continuum. If $f: X \rightarrow \mathbb{R}^{\infty}$ is an absolutely measurable function, then there is a function $g: X \rightarrow \mathbb{R}^{\infty}$ in the Baire class 1 such that $\mathrm{P}(\{x: f(x)=g(x)\})=X$.

Proof. The proof follows the lines of the proof by Bruckner, Davies and Goffman given in [4, Theorem 2.10] for the real-valued case.

With $f=\sum_{j=1}^{\infty} f_{j} e_{j}$, let $f_{j, i}$ be absolutely measurable functions that take values only from $m / 2^{i}, m=0, \pm 1, \pm 2, \ldots$, and satisfy

$$
f_{j, i}(x) \leq f_{j}(x)<f_{j, i}(x)+2^{-i} \quad \text { whenever } \quad x \in X
$$

for $j=1,2, \ldots$ and $i=0,1,2, \ldots$. For each $i$ let

$$
F_{i}=\sum_{j=1}^{i} f_{j,(i-j+1)} e_{j}+\sum_{j>i} f_{j, 0} e_{j}
$$

By Lemma 1.7 there is a function $\Phi_{0}=\sum_{j=1}^{\infty} \varphi_{j, 0} e_{j}$ in the Baire class 1 such that $\mathrm{P}\left(E_{0}\right)=X$ where $E_{0}=\left\{x: \Phi_{0}(x)=F_{0}(x)\right\}$. With the aid of Lemma 1.9, we can construct a sequence

$$
\Phi_{i}=\sum_{j=1}^{i} \varphi_{j,(i-j+1)} e_{j}+\sum_{j>i} \varphi_{j, 0} e_{j}, \quad i=1,2, \ldots,
$$

of functions in the Baire class 1 and a sequence of absolutely measurable sets $E_{i}, i=1,2, \ldots$, such that $E_{i} \subset E_{i-1}, \mathrm{P}\left(E_{i}\right)=X, F_{i}\left|E_{i}=\Phi_{i}\right| E_{i}$, and $\rho\left(\Phi_{i}(x), \Phi_{i-1}(x)\right) \leq i 2^{-i}$ whenever $x \in X$. Since $\varphi=\sum_{k=1}^{\infty}\left(\Phi_{k}-\Phi_{k-1}\right)+\Phi_{0}$ 
converges uniformly as an $\mathbb{R}^{\infty}$-valued series on $X$, we have that $\varphi$ is a Baire class 1 function and that $\Phi_{i}, i=1,2, \ldots$, converges uniformly on $X$ to $\varphi$.

It is easily shown that $\rho\left(F_{i}(x), f(x)\right) \leq(i+2) 2^{i+1}$ whenever $x \in X$. To complete the proof of the theorem we shall show that $f$ and $\varphi$ meet the requirement of Lemma 1.10. We have for each $i$

$$
(f-\varphi)\left|E_{i}=\left(f-F_{i}\right)\right| E_{i}+\left(F_{i}-\Phi_{i}\right)\left|E_{i}+\left(\Phi_{i}-\varphi\right)\right| E_{i} .
$$

The middle term on the right side of the above equation is identically 0 and the other two terms tend unifomly to 0 as $i$ tends to infinity. The theorem is proved.

\subsection{Absolute Essential Supremum Modulo a Closed Set}

Let $f$ be an absolutely measurable real-valued function defined on a continuum $X$ and let $F$ be a closed subset of $X$. The absolute essential supremum modulo $F$ of $f$ is defined to be

$$
\text { ab. ess. } \sup f=\inf \{M:\{x: f(x)>M\} \backslash F \in \operatorname{ab} \mathcal{N}(X)\}
$$

and the absolute essential infimum modulo $F$ of $f$ is defined analogously. The absolute essential norm modulo $F$ of $f$ is

$$
\text { ab }\|f\|_{\infty}=\text { ab. ess. } \sup |f| .
$$

Since the closed set $F$ will remain fixed, we shall not display the dependence on $F$ in the notations.

For absolutely measurable functions $f$ we have

$$
\text { ab. ess. } \sup f=\sup \{M:\{x: f(x)>M\} \backslash F \notin \operatorname{ab} \mathcal{N}(X)\},
$$

whence, for each $M$ with $M<$ ab. ess. sup $f$, there is a $\mu$ in $\mathrm{M}(X)$ with

$$
\mu(\{x: f(x)>M\} \backslash F)>0 .
$$

Even more, there is a $\mu$ in $\mathrm{M}(X)$ such that $\mu(\{x: f(x)>M\} \backslash F)>0$ whenever $M<$ ab. ess. $\sup f$. Indeed, if $M_{i}, i=1,2, \ldots$, is a strictly increasing sequence of real numbers that converges to ab. ess. $\sup f$, then there is a sequence $\mu_{i}, i=1,2, \ldots$, in $\mathrm{M}(X)$ such that $\mu_{i}\left(\left\{x: f(x)>M_{i}\right\} \backslash F\right)>0$ for every $i$. The measure

$$
\mu=\sum_{i=1}^{\infty}\left(2^{i} \mu_{i}(X)\right)^{-1} \mu_{i}
$$

meets the requirement. 
The usual norm properties hold for the absolute essential norm modulo $F$. For example,

$$
\begin{gathered}
\mathrm{ab}\|f\|_{\infty}=0 \quad \text { if and only if }\{x: f(x) \neq 0\} \backslash F \in \operatorname{ab} \mathcal{N}(X), \\
\text { ab }\|f+g\|_{\infty} \leq \mathrm{ab}\|f\|_{\infty}+\mathrm{ab}\|g\|_{\infty} \\
\mathrm{ab}\|f \cdot g\|_{\infty} \leq \mathrm{ab}\|f\|_{\infty} \text { ab }\|g\|_{\infty}
\end{gathered}
$$

For each $\mu$ in $\mathrm{M}(X)$ let the supremum norm of $f$ be denoted by

$$
\|f\|_{\mu, \infty}=\inf \{M:\{x:|f(x)|>M\} \in \mathcal{N}(X, \mu)\}
$$

where $\mathcal{N}(X, \mu)=\{Z: \mu(Z)=0\}$. We have

$$
\text { ab }\|f\|_{\infty} \geq\left\|f \cdot\left(1-\chi_{F}\right)\right\|_{\mu, \infty} \quad \text { whenever } \quad \mu \in \mathrm{M}(X)
$$

because $\operatorname{ab} \mathcal{N}(X) \subset \mathcal{N}(X, \mu)$ for each $\mu$ in $\mathrm{M}(X)$.

Theorem 1.12. Let $X$ be a continuum and $F$ be a closed subset of $X$. For each absolutely measurable function $f: X \rightarrow \mathbb{R}$, there is a positive measure $\mu$ such that the absolute essential norm modulo $F$ satisfies

$$
\mathrm{ab}\|f\|_{\infty}=\left\|f \cdot\left(1-\chi_{F}\right)\right\|_{\mu, \infty} .
$$

Proof. We have observed earlier the existence of a measure $\mu_{0}$ in $\mathrm{M}(X)$ such that ab $\|f\|_{\infty}=\left\|f \cdot\left(1-\chi_{F}\right)\right\|_{\mu_{0}, \infty}$. Let $\nu \in \mathrm{M}_{p}(X)$. To complete the proof we use the measure $\mu=\mu_{0}+\nu$. Indeed, we have

$$
\mathrm{ab}\|f\|_{\infty} \geq\left\|f \cdot\left(1-\chi_{F}\right)\right\|_{\mu, \infty} \geq\left\|f \cdot\left(1-\chi_{F}\right)\right\|_{\mu_{0}, \infty}=\mathrm{ab}\|f\|_{\infty}
$$

since $\mathcal{N}(X, \mu) \subset \mathcal{N}\left(X, \mu_{0}\right)$.

\subsection{Absolutely integrable functions .}

Let $X$ be a continuum. For absolutely measurable functions $f: X \rightarrow \mathbb{R}$ we use the usual notation $\|f\|_{\mu, 1}=\int_{X}|f| d \mu$. We denote the collection of all $\mu$-integrable functions by $\mathrm{I}_{1}(X, \mu)$ and define

$$
\operatorname{ab} \mathrm{I}_{1}(X)=\bigcap_{\mu \in \mathrm{M}(X)} \mathrm{I}_{1}(X, \mu) .
$$

Clearly, every function in ab $I_{1}(X)$ is absolutely measurable. (As the use of equality $\mu$-almost everywhere has been avoided in the above discussion, we do not use the notation $\mathrm{L}^{1}(X, \mu)$.) 
Let $F$ be a closed subset of $X$. The collection of all absolutely measurable functions whose absolute essential norms modulo $F$ are finite will be denoted by $\operatorname{abI}_{\infty}(X)$. For each absolutely measurable function $f$ we have

$$
\left\|f \cdot\left(1-\chi_{F}\right)\right\|_{\mu, 1}=\int_{X}\left|f \cdot\left(1-\chi_{F}\right)\right| d \mu \leq \mathrm{ab}\|f\|_{\infty} \mu(X) .
$$

Hence we have ab $\|f\|_{\infty} \geq \sup \left\{\left\|f \cdot\left(1-\chi_{F}\right)\right\|_{\mu, 1}: \mu \in \mathrm{M}(X)\right.$ with $\left.\mu(X)=1\right\}$. Indeed we have a stronger statement.

Proposition 1.13. For closed subsets $F$ of a continuum $X$, if $f: X \rightarrow \mathbb{R}$ is absolutely measurable, then the absolute essential norm modulo $F$ satisfies

$$
\mathrm{ab}\|f\|_{\infty}=\sup \left\{\left\|f \cdot\left(1-\chi_{F}\right)\right\|_{\mu, 1}: \mu \in \mathrm{M}_{p}(X) \text { with } \mu(X)=1\right\} .
$$

Proof. Let $M$ be such that $M<$ ab $\|f\|_{\infty}$. There is a positive measure $\nu$ such that the set $E=\{x:|f(x)|>M\}$ satisfies $\nu(E \backslash F)>0$. We may suppose further that $\nu(X)=1$. Then $\mu=(1-\varepsilon)(\nu(E \backslash F))^{-1} \nu\llcorner(E \backslash F)+\varepsilon \nu$, where $0<\varepsilon<1$, satisfies $\left\|f \cdot\left(1-\chi_{F}\right)\right\|_{\mu, 1}>M(1-\varepsilon)$ and $\mu(X)=1$.

Clearly the above supremum need not be a maximum. We have the following corollary.

Corollary 1.14. Let $F$ be a closed subset of a continuum $X$ and $f: X \rightarrow \mathbb{R}$ be absolutely measurable. If the absolute essential norm modulo $F$ of $f$ satisfies ab $\|f\|_{\infty}=\infty$, then $\left\|f \cdot\left(1-\chi_{F}\right)\right\|_{\mu, 1}=\infty$ for some measure $\mu$ in $\mathrm{M}_{p}(X)$.

PROOF. For each $n$ let $\mu_{n}$ be a positive measure that satisfies $\mu_{n}(X)=1$ and $\left\|f \cdot\left(1-\chi_{F}\right)\right\|_{\mu_{n}, 1}>2^{n}$. Let $\mu=\sum_{n=1}^{\infty} 2^{-n} \mu_{n}$.

We shall revisit Theorem 1.11, Proposition 1.13 and its corollary later in the context of compact manifolds.

\subsection{Lebesgue Equivalence}

For a continuum $X$ the group of self-homeomorphisms of $X$ will be denoted by $\mathcal{H}(X)$ where the group operation is composition.

Let us begin with $\mathcal{H}\left(I^{n}\right)$. According to Lebesgue [10], two functions $f$ and $g$ on $I^{n}$ are equivalent if there is an $h$ in $\mathcal{H}\left(I^{n}\right)$ such that the composition $f h$ equals $g$. In the literature this equivalence is referred to as Lebesgue equivalence of functions. (See for example $[2,3]$.)

We extend in the obvious way the definition of Lebesgue equivalence of functions to functions defined on continua. The composition $f h$ will be denoted in the contravariant notation $h^{\#} f$ since $\left(h_{1} h_{2}\right)^{\#} f=h_{2}{ }^{\#} h_{1}{ }^{\#} f$ whenever 
$h_{1} \in \mathcal{H}(X)$ and $h_{2} \in \mathcal{H}(X)$. We warn the reader that the topological notion of Lebesgue equivalence of functions differs from the measure theoretic notion of "equal $\mu$-almost everywhere."

For each measure $\mu$ on $X$ and each Borel measurable map $\varphi: X \rightarrow Y$, a measure $\varphi_{\#} \mu$ on $Y$ is induced by the formula

$$
\varphi_{\#} \mu(E)=\mu\left(\varphi^{-1}[E]\right) \text { where } E \text { is a Borel subset of } Y .
$$

It follows easily that $\left(h_{1} h_{2}\right)_{\#} \mu=h_{1 \#} h_{2 \#} \mu$ whenever $h_{1} \in \mathcal{H}(X)$ and $h_{2} \in$ $\mathcal{H}(X)$.

In a manner analogous to functions, measures $\mu$ and $\nu$ on $X$ are said to be Lebesgue equivalent measures if there is an $h$ in $\mathcal{H}(X)$ such that the induced measure $h_{\#} \mu$ is equal to $\nu$.

For measurable maps, it is well-known that inverse images of measurable sets need not be measurable. But this is not the case for absolutely measurable sets. Indeed, we have the following.

Proposition 1.15. Let $X$ be a continuum. For every measure $\mu$ in $\mathrm{M}(X)$, if $h \in \mathcal{H}(X)$ and if $E$ is an absolutely measurable set, then $h^{-1}[E]$ is $\mu$-measurable and $h_{\#} \mu(E)=\mu\left(h^{-1}[E]\right)$.

Proof. As $E$ is absolutely measurable and $h_{\#} \mu$ is Borel regular we have the two identities

$$
\begin{aligned}
& h_{\#} \mu(E)=\sup \left\{h_{\#} \mu(F): F \subset E, F \text { is closed }\right\} \\
& h_{\#} \mu(E)=\inf \left\{h_{\#} \mu(U): E \subset U, U \text { is open }\right\} .
\end{aligned}
$$

Now we have $h_{\#} \mu(F)=\mu\left(h^{-1}[F]\right)$ and $h_{\#} \mu(U)=\mu\left(h^{-1}[U]\right)$ for closed sets $F$ and open sets $U$. As $h^{-1}[F] \subset h^{-1}[E] \subset h^{-1}[U]$, the proposition follows.

Theorem 1.16. For a continuum $X$ let $h \in \mathcal{H}(X)$. Then $h^{-1}[E] \in \operatorname{ab} \mathcal{A}(X)$ whenever $E \in \operatorname{ab} \mathcal{A}(X)$ and $h^{-1}[Z] \in \operatorname{ab} \mathcal{N}(X)$ whenever $Z \in \operatorname{ab} \mathcal{N}(X)$. Consequently, $h^{\#} f$ is absolutely measurable whenever $f$ is. Indeed, for absolutely measurable, real-valued functions $f$ and for $\mu$ in $\mathrm{M}(X), h^{-1}\left[f^{-1}[E]\right]=$ $\left(h^{\#} f\right)^{-1}[E]$ holds for every Borel subset $E$ of $\mathbb{R}$, whence $h_{\#} \mu\left(f^{-1}[[a, b)]\right)=$ $\mu\left(\left(h^{\#} f\right)^{-1}[[a, b)]\right)$ holds and therefore $\int_{X} f d\left(h_{\#} \mu\right)=\int_{X}\left(h^{\#} f\right) d \mu$.

Proof. The first two conclusions follow from Proposition 1.15 above and the definitions of $\operatorname{ab} \mathcal{A}(X), \operatorname{ab} \mathcal{N}(X)$ and absolute measurability of $f$. The next statement follows from the identity $h^{-1} f^{-1}=(f h)^{-1}=\left(h^{\#} f\right)^{-1}$, and finally the definition of the integral. 


\subsection{The von Neumann-Ulam-Oxtoby Theorem}

The von Neumann-Ulam-Oxtoby Theorem concerns the Lebesgue measure $\lambda_{n}$ on intervals $I^{n}$ contained in $\mathbb{R}^{n}$. The $h_{\#} \mu$ notation for self-homeomorphisms $h$ of $I^{n}$ from the previous section will be used. We state the theorem.

Theorem (von Neumann-Ulam-Oxtoby). Let $\mu$ be a complete, Borel regular measure on the interval $I^{n}$ of $\mathbb{R}^{n}$ with $\mu\left(I^{n}\right)=\lambda_{n}\left(I^{n}\right)$, where $\lambda_{n}$ is the Lebesgue measure on $\mathbb{R}^{n}$. In order that there exists a self-homeomorphism $h$ of $I^{n}$ with $\lambda_{n}=h_{\#} \mu$, it is necessary and sufficient that $\mu$ satisfies the three conditions.

1. $\mu\left(\partial I^{n}\right)=0$.

2. $\mu$ is nonatomic.

3. $\mu(U)>0$ whenever $U$ is a nonempty, relatively open subset of $I^{n}$.

Moreover, $h$ may be assumed to be the identity map on $\partial I^{n}$.

Employing the terminology presented in the earlier Section 1.5, we find that the von Neumann-Ulam-Oxtoby Theorem exhibits necessary and sufficient conditions on a measure $\mu$ so that it is Lebesgue equivalent to the Lebesgue measure $\lambda_{n}$. The conditions are $\mu \in \mathrm{M}_{p}\left(I^{n}\right), \mu\left(\partial I^{n}\right)=0$ and $\mu\left(I^{n}\right)=\lambda_{n}\left(I^{n}\right)$. (We remind the reader that the measures in $\mathrm{M}_{p}(M)$ are nonatomic.)

The theorem was conjectured by S. M. Ulam in 1936 and was proved first (but not published) by J. von Neumann [14]. A second proof was given by Oxtoby and Ulam [12]. A third and different proof was given by Goffman and G. Pedrick [5]. A deformation version of the above theorem was established in Chapter 7 of [4]. (See Theorem 7.1 on page 90.)

\section{Manifolds}

Only compact, connected manifolds with or without boundary will be considered. The boundary of a manifold $M$ will be denoted by $\partial M$. The reader should be aware of the distinction between the boundary $\partial M$ and the boundary $\operatorname{Bd}_{M}(M)$. The second is always the empty set whereas the first need not be empty. The Brouwer Invariance of Domain Theorem [8, Theorem VI9, page 95] gives us the useful invariant $h[\partial M]=\partial M$ for every $h$ in $\mathcal{H}(M)$.

The conclusion of the von Neumann-Ulam-Oxtoby Theorem is named the von Neumann-Ulam-Oxtoby property. The purpose of the section is to prove that every manifold has this property, thereby generalizing the von NeumannUlam-Oxtoby Theorem. It is an easy matter to show that topological $n$-cells 
have the von Neumann-Ulam-Oxtoby property. A series of lemmas will show that the $n$-cell case implies the theorem for the general manifold.

\subsection{Lebesgue-Like Measures}

In view of the von Neumann-Ulam-Oxtoby Theorem we shall say that a measure $\mu$ on a compact, connected manifold $M$ is Lebesgue-like if it satisfies the conditions $\mu \in \mathrm{M}_{p}(M)$ and $\mu(\partial M)=0$. (Recall that the measures in $\mathrm{M}_{p}(M)$ are nonatomic.) For $M$, the collection of all Lebesguelike measures will be denoted by $\mathrm{M}_{L}(M)$. We have, for each $h$ in $\mathcal{H}(M)$, $h_{\#} \mu \in \mathrm{M}_{L}(M)$ whenever $\mu \in \mathrm{M}_{L}(M)$. A compact, connected manifold $M$ is said to have the von Neumann-Ulam-Oxtoby property if for each pair of measures $\mu$ and $\nu$ in $\mathrm{M}_{L}(M)$ with $\mu(M)=\nu(M)$ there is a homeomorphism $h$ in $\mathcal{H}(M)$ such that $h_{\#} \mu=\nu$ and $h \mid \partial M$ is the identity map id $\partial M$.

We have the following assertion.

Theorem 2.1. Every topological n-cell has the von Neumann-Ulam-Oxtoby property.

Proof. Let $A$ be a topological $n$-cell and let $\varphi: A \rightarrow I^{n}$ be a homeomorphism. By the von Neumann-Ulam-Oxtoby Theorem, for measures $\mu$ and $\nu$ in $\mathrm{M}_{L}(A)$ with $\mu(A)=\nu(A)$ there is a self-homeomorphism $H$ of $I^{n}$ such that $H_{\#} \varphi_{\#} \mu=\varphi_{\#} \nu$ and $H \mid \partial I^{n}$ is the identity map. Let $h=\varphi^{-1} H \varphi$. Then

$$
h_{\#} \mu=\varphi_{\#}^{-1}\left(H_{\#} \varphi_{\#} \mu\right)=\varphi_{\#}^{-1}\left(\varphi_{\#} \nu\right)=\nu
$$

and $h \mid \partial A$ is the identity map.

Observe that the exact same proof will show that the von Neumann-UlamOxtoby property is a topological invariant.

In passing, we make the following observation. A topological 2-cell $M$ has many embeddings into $\mathbb{R}^{2}$. Supppose that $M$ is an embedding such that $\lambda_{2}(\partial M)>0$ (which, of course, exists). Then the measure $\lambda_{2} \mid M$ is not Lebesgue-like on the manifold $M$. Thus we caution the reader that the notion of Lebesgue-like measures is a topological notion and yet the restiction of a Lebesgue-like measure to a submanifold may not result in a Lebesgue-like measure on the submanifold.

\subsection{Absolutely Measurable Sets of Manifolds}

We begin with the existence of nonatomic Borel regular measures associated with absolutely measurable sets $E$ of a manifold $M$. 
Proposition 2.2. Let $M$ be a compact, connected manifold and let $E$ be any absolutely measurable set. Then there exists a nonatomic Borel regular measure $\mu$ such that $\mu(U \cap E)>0$ whenever $U$ is an open set with $(U \backslash \partial M) \cap$ $\mathrm{P}(E) \neq \emptyset$ and such that $\mu((M \backslash E) \cup \partial M)=0$.

Proof. Let $U_{i}, i=1,2, \ldots$, be a basis of open sets for the topology of $M$. From the definition of the closure-like operation $\mathrm{P}$ there is a nonempty perfect set $F_{i}$ contained in $\left(U_{i} \backslash \partial M\right) \cap E$ whenever $\left(U_{i} \backslash \partial M\right) \cap \mathrm{P}(E) \neq \emptyset$. For each set $F_{i}$ let $\mu_{i}$ be a nonatomic Borel regular measure such that the topological support of $\mu_{i}$ is $F_{i}$. (See the discussion of Section 1.1.) We may assume that $\mu_{i}(M) \leq 2^{-i}$ holds. Define $\mu=\sum_{i=1}^{\infty} \mu_{i}$ to complete the proof.

Here is a useful $\mathcal{H}(M)$-invariance lemma.

Lemma 2.3. Let $h$ be in $\mathcal{H}(M)$ and let $f$ be an absolutely measurable, realvalued function on $M$, where $M$ is a compact, connected manifold. For Borel subsets $E$ of $\mathbb{R}, h^{-1}\left[f^{-1}[E] \backslash \partial M\right]=\left(h^{\#} f\right)^{-1}[E] \backslash \partial M$. Consequently, if $E$ is any Borel subset of $\mathbb{R}$ and if $\mu \in \mathrm{M}(M)$, then

$$
h_{\#} \mu\left(f^{-1}[E] \backslash \partial M\right)=\mu\left(\left(h^{\#} f\right)^{-1}[E] \backslash \partial M\right),
$$

and thereby

$$
f^{-1}[E] \backslash \partial M \in \operatorname{ab} \mathcal{N}(M) \text { if and only if }\left(h^{\#} f\right)^{-1}[E] \backslash \partial M \in \operatorname{ab} \mathcal{N}(M) .
$$

Proof. Clearly, $h^{-1} f^{-1}=(f h)^{-1}=\left(h^{\#} f\right)^{-1}$. By the Brouwer Invariance of Domain Theorem we have $h^{-1}[\partial M]=\partial M$. So the first identity holds. The rest of the lemma follows easily.

\subsection{Preliminary Lemmas}

The following lemmas will lead to the proof of the main lemma and theorem in the next section.

Lemma 2.4. Let $\mu$ and $\nu$ be Lebesgue-like measures on a compact, connected manifold $M$ and let $A$ be an $n$-cell contained in $M$ with $(\mu+\nu)(\partial A)=0$. Suppose that $U$ and $V$ are disjoint, nonempty open sets contained in $A \backslash \partial A$ with $(\mu-\nu)(U) \geq 0$. Then there is a self-homeomorphism $h$ of $M$ such that $h$ is the identity map on $(M \backslash A) \cup \partial A$ and such that

$$
h_{\#} \mu-\nu=(\mu-\nu) \mathrm{L}(M \backslash U)+\frac{(\mu-\nu)(U)}{\nu(V)} \nu\llcorner V,
$$

whence $\left(h_{\#} \mu-\nu\right)(U)=0,\left(h_{\#} \mu-\nu\right)(V)=(\mu-\nu)(U \cup V)$, and $h_{\#} \mu(Z)=0$ whenever $(\mu+\nu)(Z)=0$. 
Proof. With $\mu_{0}$ defined by

$$
\mu_{0}=\nu+(\mu-\nu)\left\llcorner(M \backslash U)+\frac{(\mu-\nu)(U)}{\nu(V)} \nu\llcorner V,\right.
$$

we have the two equations:

$$
\begin{gathered}
\mu_{0}=\nu\left\llcorner U+\mu\left\llcorner(M \backslash U)+\frac{(\mu-\nu)(U)}{\nu(V)} \nu\llcorner V,\right.\right. \\
\mu-\mu_{0}=(\mu-\nu)\left\llcorner U-\frac{(\mu-\nu)(U)}{\nu(V)} \nu\llcorner V .\right.
\end{gathered}
$$

From equation (4) we see that $\mu_{0}$ is Lebesgue-like, and from equation (5) we see that $\mu(A)=\mu_{0}(A)$ and $\mu_{0}(\partial A)=0$. Hence we may apply Theorem 2.1 to $\mu_{0} \mid A$ and $\mu \mid A$. Let $h$ be a self-homeomorphism of $A$ such that $h \mid \partial A$ is the identity map and $h_{\#}(\mu \mid A)=\mu_{0} \mid A$. Define $h$ on $M \backslash A$ to be the identity map. From $\operatorname{dim} U=\operatorname{dim} M \geq \operatorname{dim} A \geq \operatorname{dim} U$ we find that $M$ is $n$-dimensional. We infer from the Brouwer Invariance of Domain Theorem that $h$ is a selfhomeomorphism of $M$. Then $h_{\#} \mu=\mu_{0}$ as measures on $M$. The remaining assertions of the lemma follow from equation (3).

We turn to compact, connected, $n$-dimensional manifolds $M$ that are not topological $n$-cells. Let $\mathcal{C}$ be a finite collection of $n$-cells contained in $M$ with $M=\bigcup\left\{\operatorname{Int}_{M}(A): A \in \mathcal{C}\right\}$. Let $Z_{\mathcal{C}}=\bigcup\{\partial A: A \in \mathcal{C}\}$. As $M$ is not an $n$-cell we have card $\mathcal{C}>1$. A Lebesgue-like measure $\mu$ on $M$ is said to be compatible with $\mathcal{C}$ whenever $\mu\left(Z_{\mathcal{C}}\right)=0$.

Corresponding to the collection $\mathcal{C}$ is the collection of open sets $A \backslash \partial A$ and $M \backslash(A \cup \partial M), A \in \mathcal{C}$. Using this collection, we associate with $\mathcal{C}$ the finite collection $\mathcal{V}_{\mathcal{C}}$ of mutually disjoint, nonempty open sets satisfying the conditions:

$$
\begin{aligned}
& U \subset A \text { whenever } U \in \mathcal{V}_{\mathcal{C}}, A \in \mathcal{C} \text { and } U \cap A \neq \emptyset ; \\
& M \backslash Z_{\mathcal{C}}=\bigcup\left\{U: U \in \mathcal{V}_{\mathcal{C}}\right\} \\
& Z_{\mathcal{C}}=\bigcup\left\{\operatorname{Bd}_{M}(U): U \in \mathcal{V}_{\mathcal{C}}\right\} .
\end{aligned}
$$

We are now ready for the final lemma of this section.

Lemma 2.5. Suppose that $M$ is a compact, connected $n$-dimensional manifold that is not an $n$-cell. Let $\mathcal{C}$ and its associated collection $\mathcal{V}_{\mathcal{C}}$ be as described above and let $\nu$ be a Lebesgue-like measure on $M$ that is compatible with $\mathcal{C}$. If $\mu$ is a Lebesgue-like measure on $M$ such that $\mu(M)=\nu(M)$ and $\mu$ is compatible 
with $\mathcal{C}$, then there is a self-homeomorphism $h$ of $M$ such that $h \mid \partial M$ is the identity map, $h_{\#} \mu(M)=\nu(M), h_{\#} \mu$ is compatible with $\mathcal{C}$, and

$$
\operatorname{card}\left\{U: U \in \mathcal{V}_{\mathcal{C}}, h_{\#} \mu(U) \neq \nu(U)\right\}=0 .
$$

Proof. Let $k(\mathcal{C})$ be the minimum $k_{h}=\operatorname{card}\left\{U: U \in \mathcal{V}_{\mathcal{C}}, h_{\#} \mu(U) \neq \nu(U)\right\}$ where $h$ is a self-homeomorphism of $M$ satisfying the conditions $h \mid \partial M=\mathrm{id}{ }_{\partial M}$, $h_{\#} \mu(M)=\nu(M)$, and $h_{\#} \mu$ is compatible with $\mathcal{C}$.

We must prove $k(\mathcal{C})=0$. To the contrary, suppose $k(\mathcal{C})>0$. Let $h_{0}$ be such that $k_{h_{0}}=k(\mathcal{C})$. We designate by $U_{\max }$ and $U_{\min }$ the members of $\mathcal{V}_{\mathcal{C}}$ for which

$$
\left(h_{0 \#} \mu-\nu\right)\left(U_{\min }\right) \leq\left(h_{0 \#} \mu-\nu\right)(U) \leq\left(h_{0 \#} \mu-\nu\right)\left(U_{\max }\right)
$$

whenever $U \in \mathcal{V}_{\mathcal{C}}$. As $0=\left(h_{0 \#} \mu-\nu\right)(M)=\sum_{U \in \mathcal{V}_{\mathcal{C}}}\left(h_{0 \#} \mu-\nu\right)(U)$, we infer from the properties of the collection $\mathcal{V}_{\mathcal{C}}$ that

$$
\left(h_{0 \#} \mu-\nu\right)\left(U_{\max }\right)>0>\left(h_{0 \#} \mu-\nu\right)\left(U_{\min }\right) .
$$

We consider only the case where no $A$ in $\mathcal{C}$ contains both $U_{\max }$ and $U_{\min }$ since the contrary case is a simple modification of this case.

There is a finite sequence $A_{i}, i=1,2, \ldots, N$, in $\mathcal{C}$ such that $U_{\max } \subset A_{1}$, $U_{\min } \subset A_{N}$, and $\left(A_{i} \backslash \partial A_{i}\right) \cap\left(A_{i+1} \backslash \partial A_{i+1}\right) \neq \emptyset$ for each $i$. Let $U_{i}$ be a member of $\mathcal{V}_{\mathcal{C}}$ contained in $A_{i} \cap A_{i+1}$. We may suppose that $U_{\max } \neq U_{1}$, $U_{i} \neq U_{i+1}$ for all $i$, and $U_{N-1} \neq U_{\min }$. Let $i_{0}$ be the smallest integer such that $\left(h_{0 \#} \mu-\nu\right)\left(U_{i_{0}}\right) \neq 0$. Then $\left(h_{0 \#} \mu-\nu\right)\left(U_{i}\right)=0$ whenever $1 \leq i<i_{0}$. We apply Lemma 2.4, where $h_{0 \#} \mu$ replaces $\mu$, to the sets $U_{\max }$ and $U_{1}$ to get a selfhomeomorphism $h_{1}$ of $M$ such that $h_{1} \mid \partial M=\operatorname{id}_{\partial M},\left(h_{1} h_{0}\right)_{\#} \mu(M)=\nu(M)$, $\left(h_{1} h_{0}\right)_{\#} \mu$ is compatible with $\mathcal{C}$, and

$$
\begin{aligned}
\left(\left(h_{1} h_{0}\right)_{\#} \mu-\nu\right)\left(U_{\max }\right) & =0, \\
\left(\left(h_{1} h_{0}\right)_{\#} \mu-\nu\right)\left(U_{1}\right) & =\left(h_{0 \#} \mu-\nu\right)\left(U_{\max } \cup U_{1}\right)=\left(h_{0 \#} \mu-\nu\right)\left(U_{\max }\right)>0, \\
\left(\left(h_{1} h_{0}\right)_{\#} \mu-\nu\right)(U) & =\left(h_{0 \#} \mu-\nu\right)(U) \text { for } U \in \mathcal{V} \backslash\left\{U_{\max }, U_{1}\right\},
\end{aligned}
$$

whence $k_{h_{1} h_{0}}=k_{h_{0}}$. Repeating this procedure $i_{0}$ times, we have self-homeomorphisms $h_{i}$ of $M, i=1,2, \ldots, i_{0}$, such that $H=h_{i_{0}} \cdots h_{1} h_{0}$ satisfies the requirements $H \mid \partial M=\operatorname{id}_{\partial M}, H_{\#} \mu(M)=\nu(M), H_{\#} \mu$ is compatible with $\mathcal{C}$, and

$$
\begin{aligned}
\left(H_{\#} \mu-\nu\right)\left(U_{\max }\right) & =0 \\
\left(H_{\#} \mu-\nu\right)\left(U_{i_{0}}\right) & =\left(h_{0 \#} \mu-\nu\right)\left(U_{\max } \cup U_{i_{0}}\right) \\
\left(H_{\#} \mu-\nu\right)(U) & =\left(h_{0 \#} \mu-\nu\right)(U) \quad \text { for } \quad U \in \mathcal{V} \backslash\left\{U_{\max }, U_{i_{0}}\right\}
\end{aligned}
$$


whence $k_{H}<k_{h_{0}}$. Thus the contradiction $k(\mathcal{C}) \leq k_{H}<k_{h_{0}}=k(\mathcal{C})$ has appeared and the lemma is proved.

\subsection{Main Theorem}

Lemma 2.6. Suppose that $M$ is a compact, connected $n$-dimensional manifold that is not an $n$-cell. Let $\mathcal{C}$ and its associated collection $\mathcal{V}_{\mathcal{C}}$ be as described above. If $\mu$ and $\nu$ are Lebesgue-like measures on $M$ that are compatible with $\mathcal{C}$ and satisfy $\mu(M)=\nu(M)$ and card $\left\{U: U \in \mathcal{V}_{\mathcal{C}}, \mu(U) \neq \nu(U)\right\}=0$, then there is a self-homeomorphism $h$ of $M$ such that it is the identity map on $\partial M$ and such that $h_{\#} \mu=\nu$.

Proof. Index the finite set $\mathcal{C}$ as $A_{i}, i=1,2, \ldots, N$. As $\mu\left(A_{1}\right)=\nu\left(A_{1}\right)$, we infer from Theorem 2.1 that there is a self-homeomorphism $h_{1}$ of $M$ such that it is the identity map on $\left(M \backslash A_{1}\right) \cup \partial A_{1}$ and such that $\left(h_{1 \#} \mu\right)\left\llcorner A_{1}=\nu\left\llcorner A_{1}\right.\right.$. Clearly, card $\left\{U: U \in \mathcal{V}_{\mathcal{C}}, h_{1 \#} \mu(U) \neq \nu(U)\right\}=0, h_{1 \#} \mu$ is compatible with $\mathcal{C}$ and $h_{1 \#} \mu(M)=\nu(M)$. Hence $\left(h_{1 \#} \mu\right)\left(A_{2} \backslash A_{1}\right)=\nu\left(A_{2} \backslash A_{1}\right)$. There is a selfhomeomorphism $h_{2}$ of $M$ such that it is the identity map on $\left(M \backslash A_{2}\right) \cup \partial A_{2}$ and such that $\left(h_{2 \#} h_{1 \#} \mu\right)\left\llcorner A_{2}=\nu\left\llcorner A_{2}\right.\right.$ and $\left(h_{2 \#} h_{1 \#} \mu\right)\left\llcorner A_{1}=\nu\left\llcorner A_{1}\right.\right.$. Repeating this process $N$ times, we will have self-homeomorphisms $h_{i}$ of $M$ such that they are the identity maps on $\partial M$ and such that $\left(h_{N} \cdots h_{2} h_{1}\right)_{\#} \mu=\nu$.

Theorem 2.7. Every compact, connected manifold has the von NeumannUlam-Oxtoby property.

Proof. Let $\mu$ and $\nu$ be Lebesgue-like measures on the $n$-dimensional manifold $M$ with $\mu(M)=\nu(M)$. We assume that $M$ is not an $n$-cell (see Theorem 2.1). As $M$ is compact, there is a finite collection $\mathcal{C}$ of $n$-cells such that $M=\bigcup\left\{\operatorname{Int}_{M}(A): A \in \mathcal{C}\right\}$ and such that $\mu+\nu$ is compatible with $\mathcal{C}$. Combining Lemmas 2.5 and 2.6, we have a self-homeomorphism $h$ of $M$ such that it is the identity map on $\partial M$ and $h_{\#} \mu=\nu$. The theorem is proved.

\section{Lebesgue's Notion of Equivalence}

In his paper [10], Lebesgue defined the notion of equivalence of functions $f$ and $g$ by using compositions with self-homeomorphisms $h$, that is, $f=g h$. He used it in the calculus of variations to represent curves and surfaces by continuous maps. Of course not every analytical property is invariant under the "action" of self-homeomorphisms. Nonetheless, many interesting invariant properties have been investigated when the functions were defined on $[0,1]$. (Besides those mentioned in this paper there are many more related to Fourier 
series; see the many references in [4], especially those to Goffman and Waterman.) We shall extend them now to functions defined on manifolds. The key to the proofs is the von Neumann-Ulam-Oxtoby Theorem for manifolds.

\subsection{Baire Class 1}

The following theorem is related to a theorem by Bruckner, Davies and Goffman [1] (see also [4, page 17]).

Theorem 3.1. Let $M$ be a compact, connected manifold. If $f_{j}: M \rightarrow \mathbb{R}$, $j=1,2, \ldots$, are absolutely measurable functions and $\nu$ is a Lebesgue-like measure on $M$, then there is a self-homeomorphism $h$ of $M$ such that $h \mid \partial M$ is the identity map and there are functions $g_{j}, j=1,2, \ldots$, in the Baire class 1 such that $h^{\#} f_{j}$ is equal to $g_{j} \nu$-almost everywhere.

Proof. According to Theorem 1.11 there are Baire class 1 functions $g_{j, 1}$ such that

$$
\mathrm{P}\left(\bigcap_{j=1}^{\infty}\left\{x: f_{j}(x)=g_{j, 1}(x)\right\}\right)=M .
$$

Since $\bigcap_{j=1}^{\infty}\left\{x: f_{j}(x)=g_{j, 1}(x)\right\}$ is absolutely measurable, there is a Lebesguelike measure $\mu$ with $\mu\left(\bigcup_{j=1}^{\infty}\left\{x: f_{j}(x) \neq g_{j, 1}(x)\right\}\right)=0$ (see Proposition 2.2). We may assume $\mu(M)=\nu(M)$.

As $M$ has the von Neumann-Ulam-Oxtoby property, there is a self-homeomorphism $h$ such that $\mu=h_{\#} \nu$ and $h \mid \partial M$ is the identity map. Let $g_{j}=h^{\#} g_{j, 1}$. Then

$$
\nu\left(\left\{t: h^{\#} f_{j}(t) \neq g_{j}(t)\right\}\right)=\mu\left(\left\{x: f_{j}(x) \neq g_{j, 1}(x)\right\}\right)
$$

by Lemma 2.3. As $g_{j}$ is in the Baire class 1, the theorem is proved.

Bruckner, Davies and Goffman gave in [1] a proof of the theorem for one function on the interval $[0,1]$, where $\nu=\lambda_{1}$, without the use of the von Neumann-Ulam-Oxtoby Theorem. Their proof does not extend to the compact manifold case. In passing, we remark that there are functions that are not absolutely measurable for which the conclusion of the theorem holds when $\nu$ is Lebesgue measure on $[0,1]$ (see $[7,1,4]$ ). This is a result proved by W. J. Gorman III. We extend Gorman's result to manifolds.

Theorem 3.2. Let $M$ be a compact, connected manifold and $\nu$ be a Lebesguelike measure on $M$. If $f$ is a $\nu$-measurable, real-valued function on $M$ with card $f[M]<\aleph_{0}$, then there is a self-homeomorphism $h$ of $M$ such that $h \mid \partial M$ is the identity map and there is a function $g$ in the Baire class 1 such that $h^{\#} f$ is equal to $g$-almost everywhere. 
Proof. Index the finite set $f[M]$ as $y_{i}, i=1,2, \ldots, N$. Let $A_{i}$ be a Borel subset of $f^{-1}\left[y_{i}\right]$ with $\nu\left(f^{-1}\left[y_{i}\right] \backslash A_{i}\right)=0$. Then $M=\bigcup_{i=1}^{N} \mathrm{P}\left(A_{i}\right)$. We apply Propositions 1.8 and 2.2 , and the von Neumann-Ulam-Oxtoby property to complete the proof.

\subsection{Absolute Essential Supremum Modulo $\partial M$}

The closed set of the discussion in Section 1.3 will be the boundary $\partial M$ of a compact, connected $n$-dimensional manifold $M$. As we will be concerned only with Lebesgue-like measures on $M$, the Brouwer Invariance of Domain Theorem will ensure that $\mu(h[\partial M])=0$ for every self-homeomorphism $h$ and every Lebesgue-like measure $\mu$. Consequently we may assume that the absolutely measurable functions take the value 0 on $\partial M$.

We restate Theorem 1.12 in the present context of modulo $\partial M$.

Theorem 3.3. Let $M$ be a compact, connected manifold. For each absolutely measurable function $f: M \rightarrow \mathbb{R}$, there exists a Lebesgue-like measure $\mu$ such that the absolute essential norm modulo $\partial M$ satisfies ab $\|f\|_{\infty}=\|f\|_{\mu, \infty}$. Additionally, $\mu(M)=1$ may be assumed.

The proof will be left to the reader.

Theorem 3.4. Let $M$ be a compact, connected manifold. If $f: M \rightarrow \mathbb{R}$ is absolutely measurable, then the absolute essential norm modulo $\partial M$ satisfies $\mathrm{ab}\|f\|_{\infty}=\mathrm{ab}\left\|h^{\#} f\right\|_{\infty}$ for every self-homeomorphism $h$ of $M$.

Proof. The desired identity follows from Lemma 2.3 and the definition of the absolute essential norm modulo $\partial M$.

Observe that equation (2) in Lemma 2.3 yields the useful identities

$$
\left\|\left(h^{\#} f\right)\right\|_{\mu, \infty}=\|f\|_{h_{\#} \mu, \infty} \quad \text { and } \quad\left\|\left(h^{\#} f\right)\right\|_{\mu, 1}=\|f\|_{h_{\#} \mu, 1}
$$

whenever $h \in \mathcal{H}(M), \mu \in \mathrm{M}_{L}(M)$ and $f$ is absolutely measurable.

Theorem 3.5. Let $M$ be a compact, connected manifold. If $f: M \rightarrow \mathbb{R}$ is absolutely measurable and if $\nu$ is a Lebesgue-like measure on $M$, then there is a self-homeomorphism $h$ of $M$ such that $h$ is the identity map on $\partial M$ and the absolute essential norm modulo $\partial M$ satisfies ab $\|f\|_{\infty}=\left\|h^{\#} f\right\|_{\nu, \infty}$.

Proof. For every pair of Lebesgue-like measures $\mu$ and $\nu$ with $\mu(M)=\nu(M)$ there is a self-homeomorphism $h$ such that $\mu=h_{\#} \nu$ and $h \mid \partial M$ is the identity map. Select $\mu$ as in Theorem 3.3 for the function $f$. We may assume $\mu(M)=\nu(M)$. Then from equation (6) and Theorem 3.3 we have

$$
\text { ab }\|f\|_{\infty}=\|f\|_{\mu, \infty}=\|f\|_{h_{\#} \nu, \infty}=\left\|h^{\#} f\right\|_{\nu, \infty}
$$


thereby completing the proof.

\subsection{Absolutely Integrable Functions on $M$}

We translate Proposition 1.13 into statements about manifolds modulo their boundary.

Theorem 3.6. Let $M$ be a compact, connected manifold. If $f: M \rightarrow \mathbb{R}$ is absolutely measurable, then the absolute essential norm modulo $\partial M$ satisfies

$$
\mathrm{ab}\|f\|_{\infty}=\sup \left\{\left\|h^{\#} f\right\|_{\nu, 1}: h \in \mathcal{H}(M), h \mid \partial M=\operatorname{id}_{\partial M}\right\}
$$

for every Lebesgue-like measure $\nu$ with $\nu(M)=1$.

Proof. The statement is an immediate consequence of the von NeumannUlam-Oxtoby property, equation (6) and Proposition 1.13.

Theorem 3.7. Let $M$ be a compact, connected manifold. Suppose that $f$ is an absolutely measurable, real-valued function whose absolute essential norm modulo $\partial M$ satisfies ab $\|f\|_{\infty}=\infty$. Then, for each Lebesgue-like measure $\nu$ with $\nu(M)=1$, there is a self-homeomorphism $h$ of $M$ such that $h \mid \partial M$ is the identity map and $\left\|h^{\#} f\right\|_{\nu, 1}=\infty$.

Proof. By Corollary 1.14 there is a Lebesgue-like measure $\mu$ with $\mu(M)=1$ such that $\|f\|_{\mu, 1}=\infty$. Let $h$ be such that $h \mid \partial M=\operatorname{id} \partial M$ and $\mu=h_{\#} \nu$. Then $\left\|h^{\#} f\right\|_{\nu, 1}=\|f\|_{\mu, 1}=\infty$ and the theorem is proved.

Different proofs of the above theorems when $M=[0,2 \pi]$ and $\nu=\lambda_{1}$ have been given in [6] and [4, pages 160-161]. The theorems for the manifold $[0,2 \pi]$ have applications in certain questions of "change of variables" in Fourier series. Of course the manifold in the study of Fourier series is $\mathbb{S}_{1}$.

In summary, we have established the following.

Theorem 3.8. Let $\nu$ be a Lebesgue-like measure on a compact, connected manifold $M$. For absolutely measurable, real-valued functions $f$ on $M$, a necessary and sufficient condition for the $\nu$-integrability of $h^{\#} f$ for every $h$ in $\mathcal{H}(M)$ with $h \mid \partial M=\mathrm{id}_{\partial M}$ is that $\mathrm{ab}\|f\|_{\infty}$ modulo $\partial M$ be finite.

\subsection{Product Measures}

The original von Neumann-Ulam-Oxtoby Theorem concerned the Lebesgue measure $\lambda_{n}$. Of course, $\lambda_{n}$ is a product measure. We have the following. 
Theorem 3.9. Let $\mu$ be a Lebesgue-like measure on a compact, connected manifold $M$. If $\nu$ is a Lebesgue-like measure on $M \times[0,1]$ with $\nu(M \times[0,1])=$ $\mu(M)$, then there is a self-homeomorphism $h$ of $M \times[0,1]$ such that it is the identity map on $\partial(M \times[0,1])$ and such that $h_{\#} \nu=\mu \times \lambda_{1}$, where $\lambda_{1}$ is Lebesgue measure on $[0,1]$.

As $\mu \times \lambda_{1}$ is Lebesgue-like on $M \times[0,1]$, the proposition is a trivial consequence of the von Neumann-Ulam-Oxtoby property.

\section{$3.5 \quad \mathbf{L}^{1}$-Spaces}

The von Neumann-Ulam-Oxtoby property of compact, connected manifolds $M$ has rather nice consequences for $\mathrm{L}^{1}(M, \mu)$ when $\mu$ is a Lebesgue-like measure.

Lemma 3.10. Suppose that $\mu$ is Lebesgue-like measure on a compact, connected manifold $M$. If $h$ is a self-homeomorphism of $M$ with $h \mid \partial M=\mathrm{id} \partial M$, then $\mathrm{L}^{1}(M, \nu)=\left\{h^{\#} f: f \in \mathrm{L}^{1}\left(M, h_{\#} \nu\right)\right\}$ and $\int_{M} h^{\#} f d \nu=\int_{M} f d\left(h_{\#} \nu\right)$ whenever $f \in \mathrm{L}^{1}\left(M, h_{\#} \nu\right)$.

Proof. With $\mu=h_{\#} \nu$, let $f \in \mathrm{L}^{1}(M, \mu)$ (of course, $f$ is extended realvalued). Select a Borel measurable function $g: M \rightarrow \mathbb{R}$ such that $\mu(Z)=0$ where $Z=\{x: f(x) \neq g(x)\}$. From $\left(h^{\#}(f-g)\right)^{-1}=h^{-1}(f-g)^{-1}$ we have $h^{-1}[Z]=\left\{t: h^{\#} f(t) \neq h^{\#} g(t)\right\}$. Let us show $\nu\left(h^{-1}[Z]\right)=0$. To this end, let $E$ be a Borel set such that $E \supset Z$ and $\mu(E)=0$. Then we have

$$
0=\mu(E)=h_{\#} \nu(E)=\nu\left(h^{-1}[E]\right) \geq \nu\left(h^{-1}[Z]\right) .
$$

Consequently, $h^{\#} f=h^{\#} g \nu$-almost everywhere on $M$. Since $g$ is absolutely measurable, we finally have

$$
\int_{M}\left|h^{\#} f\right| d \nu=\int_{M}\left|h^{\#} g\right| d \nu=\int_{M}|g| d\left(h_{\#} \nu\right)=\int_{M}|g| d \mu=\int_{M}|f| d \mu<\infty .
$$

Hence the inclusion $\mathrm{L}^{1}(M, \nu) \supset\left\{h^{\#} f: f \in \mathrm{L}^{1}(M, \mu)\right\}$ follows. From this we infer $\mathrm{L}^{1}(M, \mu) \supset\left\{\left(h^{-1}\right)^{\#} f: f \in \mathrm{L}^{1}(M, \nu)\right\}$. Consequently

$$
\begin{aligned}
\mathrm{L}^{1}(M, \nu) & \supset\left\{h^{\#} f: f \in \mathrm{L}^{1}(M, \mu)\right\} \\
& \supset\left\{h^{\#}\left(h^{-1}\right)^{\#} f: f \in \mathrm{L}^{1}(M, \nu)\right\}=\mathrm{L}^{1}(M, \nu)
\end{aligned}
$$

and the lemma is proved.

We have the following application. 
Theorem 3.11. Suppose that $\nu$ is a Lebesgue-like measure on a compact, connected manifold $M$ and that $F$ is a $\nu$-measurable, extended real-valued function that is real-valued $\nu$-almost everywhere on $M$. Let $\mathcal{V}$ be the vector space of all $\nu$-measurable, extended real-valued functions $f$ on $M$ with the property that $|f| \leq k F \nu$-almost everywhere on $M$ for some real number $k$. Then there exists a self-homeomorphism $h$ of $M$ such that $h \mid \partial M=\mathrm{id}_{\partial M}$ and

$$
h^{\#} f \in \mathrm{L}^{1}(M, \nu) \text { whenever } f \in \mathcal{V} .
$$

Proof. Let $\mu$ be the measure on $M$ given by $\mu(E)=\alpha \int_{E}(1+F)^{-1} d \nu$ for $E \in \mathcal{A}(M, \nu)$, where $\alpha$ is such that $\mu(M)=\nu(M)$. As $F$ is real-valued $\nu$-almost everywhere on $M$, we have that $\mu$ is Lebesgue-like. By Theorem 2.7, there is a self-homeomorphism $h$ of $M$ such that $h \mid \partial M=\operatorname{id} \partial M$ and $\mu=h_{\#} \nu$. Since $\mathcal{V} \subset \mathrm{L}^{1}(M, \mu)$ clearly holds, the theorem follows.

Corollary 3.12. Suppose that $\nu$ is a Lebesgue-like measure on a compact, connected manifold $M$. If $f_{j}, j=1,2, \ldots$, is a sequence of $\nu$-measurable, extended real-valued functions on $M$ such that $\sum_{j=1}^{\infty}\left|f_{j}\right|$ is real-valued $\nu$-almost everywhere on $M$, then there is a self-homeomorphism $h$ of $M$ such that $h \mid \partial M=\mathrm{id}_{\partial M}$ and such that $h^{\#} f_{j}$ is $\nu$-integrable for every $j$.

Proof. Let $F$ be $\sum_{j=1}^{\infty}\left|f_{j}\right|$ in the above theorem.

We have the simple corollary.

Corollary 3.13. If $f_{j}, j=1,2, \ldots$, are Lebesgue measurable, extended realvalued functions on $\mathbb{R}^{n}$ with $\sum_{j=1}^{\infty}\left|f_{j}\right|$ real-valued $\lambda_{n}$-almost everywhere, then there is a self-homeomorphism $h$ of $\mathbb{R}^{n}$ such that $h^{\#} f_{j}$ is locally Lebesgue integrable for each $j$.

Obviously, local integrability cannot be strengthened to integrability on $\mathbb{R}^{n}$. For the proof, use the compact manifolds $\{x: k-1 \leq\|x\| \leq k\}, k=1,2, \ldots$. In [13], the above corollary was proved by Świątkowski for only a single function defined on $I^{n}$ but with a stronger conclusion. (See also [9].)

Theorem 3.14. Let $\mu$ and $\nu$ be Lebesgue-like measures on a compact, connected manifold $M$ with $\mu(M)=\nu(M)$. Then there is a self-homeomorphism $h$ of $M$ such that $h \mid \partial M=\mathrm{id}_{\partial M}$ and such that $h^{\#}: \mathrm{L}^{1}(M, \mu) \rightarrow \mathrm{L}^{1}(M, \nu)$ is a norm-preserving linear isomorphism. Moreover, $h^{\#}$ preserves the Baire classes of functions.

Proof. By Theorem 2.7, there is a self-homeomorphism $h$ of $M$ such that $h \mid \partial M=\operatorname{id}_{\partial M}$ and $\mu=h_{\#} \nu$. Then the above lemma provides an onto map. 
Clearly, $h^{\#}$ is a linear map that satisfies $\|f\|_{\mu, 1}=\left\|h^{\#} f\right\|_{\nu, 1}$. That $h^{\#}$ is one-to-one follows from $\nu=\left(h^{-1}\right)_{\#} \mu$. Finally, for each $\alpha$ and any function $f$ on $M$, the function $h^{\#} f$ is in the Baire class $\alpha$ if and only if $f$ is.

\section{References}

[1] A. M. Bruckner, R. O. Davies, and C. Goffman, Transformations into Baire 1 functions, Proc. Amer. Math. Soc. 67 (1977), 62-66.

[2] L. Cesari, Recent results in surface area theory, Amer. Math. Monthly 66 (1959), 173-192.

[3] C. Goffman, Lebesgue equivalence, Real Analysis Exchange 3 (1977-78), $7-23$.

[4] C. Goffman, T. Nishiura, and D. Waterman, Homeomorphisms in analysis, Math. Surveys Monogr., Number 54, American Mathematical Society, Providence, 1997.

[5] C. Goffman and G. Pedrick, A proof of the homeomorphism of LebesgueStieltjes measure with Lebesgue measure, Proc. Amer. Math. Soc. 52 (1975), 196-198, MR 51, \#13170.

[6] C. Goffman and D. Waterman, A characterization of the class of functions whose Fourier series converge for every change of variable, J. London Math. Soc. 10 (1975), 69-74.

[7] W. J. Gorman III, Lebesgue equivalence to functions of the first Baire class, Proc. Amer. Math. Soc. 17 (1966), 831-834.

[8] W. Hurewicz and H. Wallman, Dimension theory, Princeton University Press, Princeton, 1948.

[9] B. Koszela, Tadeusz Światowski - obituary, Real Analysis Exchange 20 (1994-95), 3-5.

[10] H. Lebesgue, Intégrale, longueur, aire, Ann. Mat. Pura Appl. 7 (1902), 231-359.

[11] J. C. Oxtoby, Measure and category, second ed., Grad. Texts in Math., Springer-Verlag, New York, 1980.

[12] J. C. Oxtoby and S. M. Ulam, Measure-preserving homeomorphisms and metrical transitivity, Ann. of Math. (2) 42 (1941), 874-920. 
[13] T. Świątowski, Sur une transformation d'une fonction mesurable en une fonction sommable, Fund. Math. 52 (1963), 1-12.

[14] J. von Neumann, Collected works, Vol II: Operators, ergodic theory and almost periodic functions in a group, Pergamon, New York, 1961, p. 558. 